\title{
La nueva gripe A (H1N1) 2009 en Atención Primaria: ¿qué nos hemos encontrado?
}

\author{
P. Aizpurua Galdeano ${ }^{a}, A$. Estabanell Buxób ${ }^{\text {, R. Besora Anglerill, }}$, \\ JM. Casanovas Gordóc, N. Torner Gracia ${ }^{d}$, T. Pumarola Suñé \\ 'Pediatra. ABS 7 La Salut. Badalona, Barcelona. España. \\ ${ }^{b}$ Pediatra. ABS Gòtic (Anexo Rull). Barcelona. España. \\ 'Pediatra. CAP Roquetes-Canteres. Barcelona. España. \\ 'Direcció General de Salut Pública. Departament de Salut. Generalitat de Catalunya. Barcelona. España. \\ eServicio de Microbiología (CDB). Hospital Clínic. Barcelona. España.
}

\section{Resumen}

Objetivo: describir las características clínicas de los casos de gripe pandémica A (H1N1) 2009 diagnosticados por pediatras centinela del programa PIDIRAC (Programa de información diaria de las infecciones respiratorias agudas de Catalunya) en atención primaria (AP).

Material y métodos: la muestra de estudio estaba formada por los casos visitados por cuatro de los pediatras centinela que participan en el programa PIDIRAC. Se recogieron de forma sistemática, para evitar sesgo de muestreo, muestras faríngeas y nasales de los casos sospechosos de gripe para su estudio virológico. Cada semana del periodo de estudio se seleccionaron los dos primeros pacientes que acudieron a la consulta y cumplían con los criterios de caso sospechoso, se recogieron sus datos demográficos y clínicos y se cursó el estudio viral. A los 55 niños con gripe pandémica A (H1N1) 2009 se les realizó un seguimiento telefónico para conocer la evolución del cuadro.

Resultados: la mayor parte de los casos tenían una edad entre cinco y 14 años (89,1\%; intervalo de confianza del 95\% [IC 95\%]: 77,8-95,9). Los síntomas más frecuentes fueron la fiebre (criterio de inclusión), que duró una media de 3,6 días (desviación estándar [DE]: 1,6) y la tos. Más de la mitad refirieron también odinofagia, rinitis o cefalea. En general, el cuadro clínico fue leve, aunque un niño fue ingresado durante 48 horas por presentar una neumonía. De los 13 niños que tenían antecedentes de asma, dos presentaron exacerbaciones leves. Ningún niño recibió tratamiento antiviral.

Conclusión: a pesar de los temores iniciales, los casos de gripe pandémica A (H1N1) 2009 en los niños atendidos en nuestras consultas de AP han sido generalmente leves.

Palabras clave: Virus de la Influenza A, subtipo H1N1. Gripe humana: complicaciones. Lactante. Niño. Adolescente. Atención Primaria de salud.

Pilar Aizpurua Galdeano, 19353pag@gmail.com

Los autores declaran no presentar conflictos de intereses en relación con la preparación y publicación de este artículo. 
New influenza A (H1N1) 2009 in Primary Care: what have we found?

Abstract

Objective: to describe the clinical features of pandemic influenza cases diagnosed in primary health care by sentinel pediatricians who participate in the PIDIRAC surveillance scheme (Programa de información diaria de las infecciones respiratorias agudas de Catalunya).

Methods: study sample included the cases of confirmed influenza A (H1N1) 2009 attended by sentinel pediatricians that collaborate with PIDIRAC. Pharyngeal and nasopharyngeal swabs for virologic analysis of suspected influenza cases were collected on a systematic basis in order to avoid bias. Every week during the study period the first two patients that consult and comply with the definition of suspicious case of influenza were recruited, if consent was obtained. Demographic characteristics and clinical features were recorded, and virologic studies were performed. The fifty-five children with confirmed pandemic influenza A (H1N1) 2009 were contacted by telephone in order to obtain information regarding their clinical status.

Results: most of the cases were 5-14 years old children and adolescents $(89.1 \%, 95 \% \mathrm{Cl}$ 77.8-95.9). The most frequent symptoms were fever (inclusion criteria), that lasted a mean of 3.6 days $(S D=1.6)$ and cough. More than $50 \%$ of patients also presented with sore throw, rhinitis or headache. In general, the disease was mild even though one child was hospitalised during 48 hours because he (or she) had a pneumonia. Of the thirteen patients who suffered from asthma only two had mild exacerbations. None of the patients needed antiviral treatment.

Conclusions: despite initial fears, the cases of pandemic influenza A (H1N1) 2009 treated in our community health centres have been generally mild.

Key words: Influenza A Virus, H1N1 Subtype. Influenza, Human/complications. Infant. Child. Adolescent. Primary Health Care.

\section{Introducción}

Los primeros casos de gripe pandémica se detectaron en Estados Unidos y México en abril de $2009^{1,2}$, y a partir de entonces fueron apareciendo casos esporádicos en Europa. El 11 de junio de 2009 la OMS elevó el nivel de alerta de pandemia de la fase 5 a la fase 6 , indicando que ya estaba en marcha una pandemia mundial ${ }^{3}$. El día 8 de septiembre de 2009 se convocó una reunión de los médicos centinela del programa PIDIRAC para preparar el periodo de vigilancia de la gripe 2009-2010, que se extendía desde la semana número 40 de 2009 a la semana 20 de $2010^{4}$. De forma excepcional, el pasado año el programa había funcionado también durante el verano debido a la amenaza de la gripe pandémica. El primer caso de aislamiento del virus de la gripe pandémica A (H1N1) 2009 en Atención Primaria (AP) por parte de los médicos centinela desde el inicio de la pandemia tuvo lugar en una de nuestras consultas en junio de 2009. Era una niña de siete años, nacida en Colombia pero residente en España desde hacía cinco años, sin antecedentes de viajes a su país o contacto conocido con personas proceden- 
tes de zonas de riesgo. Posteriormente, se fueron aislando de manera esporádica más casos, algunos relacionados con asistencia a colonias infantiles. Algunos pediatras de AP que participamos como centinelas consideramos que sería de interés describir las característica clínicas de los casos de gripe pandémica que podríamos identificar gracias al programa PIDIRAC.

\section{Material y métodos}

El sistema de vigilancia de la gripe de Catalunya estaba formado en el año 2009 por 51 médicos centinela de AP, de los cuales 24 eran pediatras. El programa cubre a una población total de 64060 (el 0,86\% de la población catalana $)^{4}$. Dicho programa recomienda que cada médico participante seleccione dos pacientes a la semana, de lunes a miércoles preferentemente, entre aquellos que se presentan con síndromes gripales. A cada paciente se le recoge un frotis faríngeo y uno nasal, que se introducen inmediatamente en un medio de transporte y se envían en menos de 24 horas al laboratorio del Hospital Clínico de Barcelona, donde se analizan las muestras mediante inmunofluorescencia indirecta (IFI), reacción en cadena de la polimerasa (PCR) y cultivo celular (CC). Los resultados se remiten directa- mente a la Subdirección General de Vigilancia Epidemiológica y Respuesta a Emergencias de Salud Pública del Departamento de Salud de la Generalitat de Cataluña, que coordina el PIDIRAC. Los resultados de las muestras se remiten a los médicos centinela en un plazo variable, siendo el máximo de 20 días para el CC negativo.

Nuestra muestra está formada por 55 niños de uno a 14 años, visitados por cuatro pediatras de AP de la red centinela PIDIRAC, que presentaron una gripe pandémica confirmada mediante estudios virológicos. Las consultas de AP están situadas en zonas urbanas de Barcelona y Badalona, y en ellas se atiende a 4000 niños, aproximadamente.

Según la definición de caso de la Unión Europea, consideramos un caso sospechoso de gripe si los niños presentaron un cuadro de inicio brusco con alguno de los síntomas siguientes: fiebre $\geq 37,8{ }^{\circ} \mathrm{C}$, cefalea, mialgia y malestar general, y alguno de los siguientes signos o síntomas de infección respiratoria aguda: tos, dificultad respiratoria, rinorrea u odinofagia ${ }^{5}$. Para evitar el sesgo de muestreo utilizamos un método de obtención de muestras sistemático, tal como sugieren los protocolos de los CDC (Centers for Disease Control and Prevention) y del Ministerio de Sanidad 
y Política Social ${ }^{6,7}$. Para ello, recogimos para el estudio, de forma sistemática, a los dos primeros pacientes que a lo largo de la semana acudieran a la consulta, cumplieran los criterios de inclusión y dieran su consentimiento a la recogida de muestras (ellos y/o sus padres o acompañantes). En el momento de recogida de los frotis se recogió también información sobre las características demográficas y el cuadro clínico que presentaban los niños. Posteriormente, al recibir el resultado virológico (7-10 días después), se contactó telefónicamente con las familias y se interrogó sobre la evolución que había seguido el cuadro gripal, las complicaciones surgidas y los tratamientos realizados. En caso de persistencia de los síntomas, se contactó de nuevo con posterioridad y se revisaron las historias clínicas de los pacientes.

Dado que la decisión de realizar el estudio se tomó en el mes de septiembre, los casos de gripe pandémica que se habían detectado previamente -desde el mes de junio- no se incorporaron a la muestra si los pacientes no habían sido seleccionados de forma sistemática. Sí se les tuvo en cuenta, sin embargo, para describir el ritmo de aparición de todos los casos de gripe pandémica A (H1N1) 2009 que se detectaron en las consultas y su distribución temporal, así como pa- ra calcular los porcentajes de positividad para gripe pandémica del total de muestras recogidas.

\section{Análisis estadístico}

Los datos se incorporaron a un archivo Excel $^{\circledast}$ y se analizaron con el programa SPSS $^{\circledR}$ versión 17. Las variables continuas se resumen como medias y se acompañan de su desviación estándar (DE) y del rango. Las variables categóricas se presentan como porcentajes y se presentan los intervalos de confianza del 95\% (IC 95\%). Se realizó un análisis de regresión logística para conocer si alguna característica demográfica de los pacientes (edad, sexo, etnia, número de convivientes/hermanos) o de la clínica de inicio (comienzo brusco, temperatura máxima, vómitos) se relacionaba con un cuadro febril más prolongado (> 5 días), estableciendo el nivel de significación estadística en un error $=0,05$.

\section{Resultados}

\section{Características demográficas y antecedentes (tabla 1)}

La mayor parte de los casos tenían una edad entre cinco y 14 años (89,1\%; IC 95\%: 77,8-95,9). Aunque pueda llamar la atención el alto número de casos en niños inmigrantes, estos datos con- 
Tabla 1. Caracteristicas de los pacientes que presentaron gripe pandémica A (H1N1) 2009 confirmada $(N=55)$

\begin{tabular}{|c|c|}
\hline \multirow[t]{2}{*}{ Sexo } & Masculino: 54,5\% (IC 95\%: 40,6-68,0) \\
\hline & Femenino: 45,5\% (IC 95\%: 32,0-59,4) \\
\hline \multirow[t]{2}{*}{ Edad en años } & Media: $7,80(\mathrm{DE}: 3,22)$ \\
\hline & Rango: $1,57-14,99$ \\
\hline \multirow[t]{2}{*}{ Grupo de edad } & 0-4 años: 10,9\% (IC 95\%: 4,1-22,2) \\
\hline & 5-14 años: 89,1\% (IC 95\%: 77,8-95,9) \\
\hline \multirow[t]{5}{*}{ Etnia } & Nacionales: $69,1 \%$ (IC 95\%: 55,2-80,9) \\
\hline & Asiáticos: 12,7\% (IC 95\%: 5,3-24,5) \\
\hline & Magrebíes: 5,5\% (IC 95\%: 1,1-15,1) \\
\hline & Iberoamericanos: 7,3\% (IC 95\%: 2,0-17,6) \\
\hline & Otros: $5,5 \%$ (IC $95 \%: 1,1-15,1)$ \\
\hline N. ${ }^{\circ}$ de convivientes en el hogar & Media: 4,11 (DE: 1,20$)$ \\
\hline N. ${ }^{\circ}$ de hermanos (incluido el paciente) & Media: 1,65 (DE: 0,98) \\
\hline \multirow[t]{3}{*}{ Patología de riesgo } & Ninguna: $74,5 \%$ (IC 95\%: 61,0-85,3) \\
\hline & Asma: $23,6 \%$ (IC 95\%: 13,2-37,0) \\
\hline & Déficit de IgA: 1,8\% (IC 95\%: 0,05-9,7) \\
\hline \multirow[t]{2}{*}{ Contacto conocido con caso de gripe A (H1N1) } & No: $94,5 \%$ (IC $95 \%: 84,9-98,9)$ \\
\hline & Sí: 5,5\% (IC 95\%: 1,1-15,1) \\
\hline
\end{tabular}

Tabla 2. Características clínicas (datos recogidos en la primera consulta, coincidente con la recogida de muestras) $(\mathrm{N}=55)$

\begin{tabular}{l}
\hline Comienzo brusco \\
\hline Temperatura máxima \\
\hline Odino $)$ \\
\hline Odinofagia \\
\hline Rinitis \\
\hline Conjuntivitis \\
\hline Otalgia \\
\hline Dolor abdominal \\
\hline Dolor muscular \\
\hline Cefalea \\
\hline Vómitos \\
\hline Diarrea \\
\hline Dificultad respiratoria \\
\hline
\end{tabular}

$85,5 \%($ IC $95 \%: 73,3-93,5)$

Media: 38,8 (DE: 0,59)

Rango: $(37,8-40,0)$

Leve $43,6 \%$ (IC 95\%: 30,3-57,7)

Moderada: 41,8 (IC 95\%: $28,7-55,9)$

Importante: 14,5 (IC 95\%: 6,5-26,7)

$65,5 \%$ (IC 95\%: 51,4-77,8)

$60,0 \%$ (IC 95\%: 45,9-73,0)

$7,3 \%($ IC $95 \%: 2,0-17,6)$

9,1\% (IC 95\%: 3,0-20,0)

$18,2 \%$ (IC 95\%: 9,1-30,9)

$29,6 \%$ (IC 95\%: 18,0-43,6)

$55,6 \%$ (IC 95\%: 41,4-69,1)

$23,6 \%$ (IC 95\%: 13,2-37,0)

$9,1 \%($ IC $95 \%: 3,0-20,0)$

$0 \%($ IC $95 \%: 0,0-6,0)$

DE: desviación estándar. IC 95\%: intervalo de confianza del 95\%.

cuerdan con las características de las consultas de Pediatría que participaron en el estudio (barrios con alta proporción de población inmigrante). Solo un 
5,5\% (IC 95\%: 1,1-15,1) de los niños con gripe había tenido contacto conocido con un caso de gripe pandémica $A$ (H1N1) 2009. El 74,5\% (IC 95\%: 61,0$85,3)$ de los niños no tenía patología de base que pudiera considerarse de riesgo para padecer una gripe grave. La patología considerada de riesgo más frecuente fue el asma (23,6\%; IC 95\%: 13,2-37,0), leve en la mayoría de los casos. Un niño tenía hipogammaglobulinemia $A$.

\section{Características clínicas (tabla 2)}

La temperatura superior a $37,8^{\circ} \mathrm{C}$ se consideró como criterio de inclusión, por lo que todos los niños que presentamos tuvieron fiebre. La media de la temperatura máxima fue de $38,8^{\circ} \mathrm{C}$ (DE: 0,59 ), con un rango de $37,8-40^{\circ} \mathrm{C}$. Todos los niños refirieron tos. Esta fue leve en el $43,6 \%$ de los casos (IC 95\%: $30,3-57,7)$. Otros signos frecuentes fueron la odinofagia $(65,5 \%$; IC $95 \%$ : $51,4-77,8)$, la rinitis $(60,0 \%$; IC 95\%: $45,9-73,0)$ y la cefalea $(55,6 \%$; IC $95 \%: 41,4-69,1)$.

\section{Complicaciones}

De los 55 niños, dos requirieron ingreso hospitalario (3,6\%; IC 95\%: 0,4$12,5)$. Uno de ellos ingresó durante 48 horas por presentar una neumonía.
El segundo niño ingresó en observación por la aparición de petequias. En este segundo caso no hubo complicaciones.

Además de los dos niños citados, dos niños más presentaron complicaciones relacionadas con el cuadro gripal (otitis y sinusitis, respectivamente) en los siguientes 15 días.

Del total de pacientes, siete $(12,7 \%$; IC 95\%: 5,3-24,4) tomaron antibióticos en los 15 días siguientes. Además de los citados, un niño presentó una amigdalitis pultácea. Los tres restantes recibieron antibiótico fuera del ámbito de la consulta de AP sin que constase un diagnóstico claro.

\section{Evolución}

Al recibir los resultados positivos para gripe pandémica A (H1N1) 2009 se contactó con las familias para informar del hecho y preguntar acerca de la evolución clínica. Basándonos en sus respuestas, la fiebre duró entre uno y siete días (media de 3,6 días; DE: 1,55) y la tos entre dos y 20 días (media de 8,5 días; DE: 3,85). En el análisis de regresión logística, ninguno de los factores estudiados se relacionó con un cuadro febril prolongado.

\section{Distribución temporal (figura 1)}

El 15 de junio se detectó el primer caso de gripe pandémica $A(\mathrm{H} 1 \mathrm{~N} 1) 2009$. 
Figura 1. Distribución temporal de los casos de gripe pandémica A (H1N1) 2009.

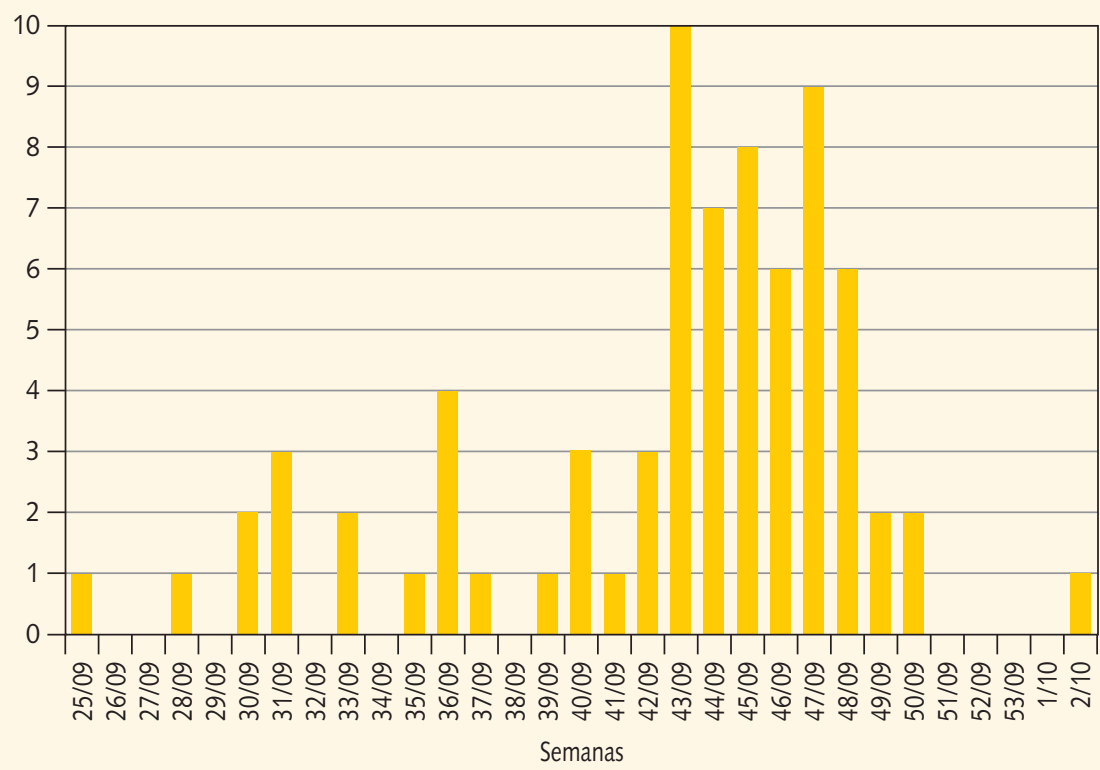

Figura 2. Porcentaje de positividad de las muestras recogidas para gripe pandémica A (H1N1) 2009.

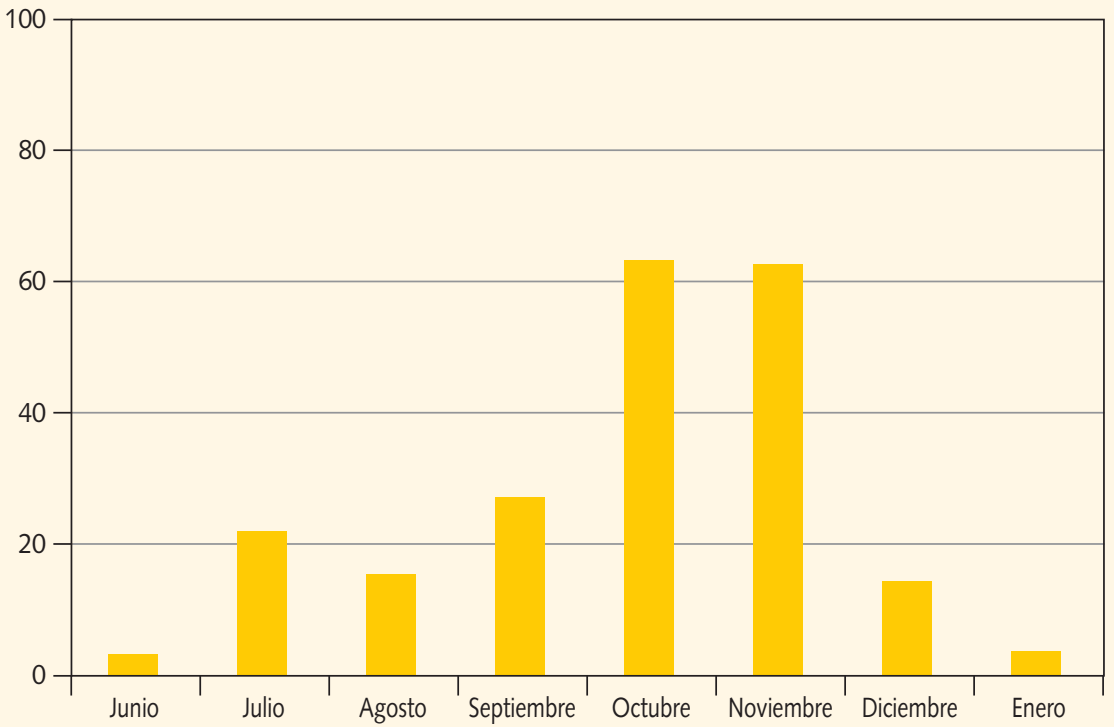


Aunque durante el verano hubo un goteo de casos, el mayor número de gripes ocurrió entre las semanas 42 y 50 del año 2009 (entre el 12 de octubre y el 13 de diciembre). Después de la semana 50 solo se ha detectado un caso más (el día 12 de enero), a pesar de que la recogida de muestras ha continuado hasta la preparación de este artículo (30 de abril). La distribución de los casos coincide con la de la totalidad de los casos de Cataluña publicada por el programa PIDIRAC ${ }^{8}$. Durante los dos meses de máxima incidencia (octubre y noviembre) el porcentaje de positividad de las muestras para gripe respecto al total de muestras remitidas se acercó al 70\% (figura 2).

\section{Discusión}

Las pandemias gripales existen desde hace siglos, aunque la primera reconocida es la del año 1580. Durante los siglos XVIII y XIX, según diferentes autores, se produjeron entre siete y ocho pandemias. En el siglo XX se declararon tres pandemias de gripe por la aparición de variantes antigénicas. Estas fueron: en los años 1918-19 la llamada gripe española, con 20-50 millones de muertes; en los años 1957-58 la gripe asiática, que provocó más de un millón de muertes; y en los años 1968-69 la gripe de Hong
Kong, que ocasionó cifras superiores a un millón de muertes. En los primeros años del siglo XXI aparecieron el síndrome de distrés respiratorio agudo grave (SRAS o SARS) en el año 2002, que causó alrededor de 8422 afectados, con 916 muertes, más de la mitad de las cuales ocurrieron en China, y el virus de la gripe A (H5N1) o cepa aviar en 1997, que se hizo más patente a partir del año 2003. La gripe aviar, epidemia animal conocida desde hace años que raras veces afectaba al hombre, hizo predecir una pandemia mundial de influenza. En diciembre de 2007 se habían registrado 438 casos declarados, con 262 muertes en el sudeste asiático, una letalidad superior al 50\% ${ }^{9-12}$. En el ámbito médico se esperaba la aparición de una nueva pandemia gripal antes o después, ya que hacía 40 años que no ocurría y cada temporada se advertía que aquella sería la definitiva.

Las epidemias que ocurren prácticamente cada invierno afectan a un elevado porcentaje de la población general provocando una elevada morbilidad y mortalidad entre aquellos que presentan factores de riesgo de complicaciones. En España, la gripe estacional ocasionó una media anual de 1400 muertes entre los años 1982 y 1999, con un pico máximo de 3185 en la temporada 1998-99, y 
aún de 543 en el año $2005^{13}$. Se consideraba que la aparición de una cepa gripal nueva que se propagara fácilmente y tuviese una alta letalidad sería una temible amenaza para la humanidad.

El primer caso de infección por el virus gripal A (H1N1) 2009 apareció durante el mes de abril en México. El 11 de junio de 2009, la Organización Mundial de la Salud declaró la pandemia, que consistía en la propagación del virus gripal por todo el mundo.

Debíamos y tuvimos que estar preparados y considerar la posibilidad de la peor situación. En el hemisferio norte apareció la enfermedad ya durante los meses estivales, lo cual nos enseñó que el virus gripal puede seguir circulando durante el verano, a pesar de las altas temperaturas alcanzadas. El hecho de seguir el control de la enfermedad gripal mediante el programa PIDIRAC durante estos meses nos permitió detectar los primeros enfermos por gripe pandémica A (H1N1) 2009.

La etapa previa al inicio escolar, durante el mes de agosto, provocó temor ante la posibilidad de que la afectación fuera general a toda la población, ya que se desconocía la gravedad que podría alcanzar la enfermedad y la morbimortalidad que conllevaría. La experiencia en los países del hemisferio sur, afectos por la epidemia durante los meses de mayo hasta septiembre, sin embargo, podía ser tranquilizadora ${ }^{14}$.

Tras unos meses de haber acabado esta epidemia en nuestro país, donde se extinguió a principios de enero contrariamente a lo pronosticado, pudimos observar que a quien afectó fundamentalmente fue a los pacientes pediátricos de cuatro a 15 años $y$, en menor medida, a los niños de cero a cuatro años. Esto se reproduce en la cohorte de nuestros pacientes diagnosticados clínicamente y confirmados virológicamente en las cuatro consultas de AP. La afectación de adultos hasta los 65 años fue menor, y prácticamente inexistente entre los mayores de 65 años de edad ${ }^{8}$.

Nuestra participación en el programa PIDIRAC nos ha dado la oportunidad de seguir la pandemia en nuestro medio desde su inicio, en verano de 2009, hasta su final, en enero de 2010 y de poder describir la clínica de los casos de gripe de nuestras consultas de Pediatría de AP. Todos los pacientes tenían como sintomatología, evidentemente, la fiebre y algún síntoma respiratorio, ya que eran los criterios de inclusión. Sin embargo, algunos estudios han descrito que entre un $8 \%$ y un $32 \%$ de los infectados presentaron cuadros afebriles ${ }^{15-17}$. En nuestros pacientes la tos, la odinofa- 
gia, la rinitis, y la cefalea fueron los síntomas más llamativos. Menos frecuentes fueron el dolor muscular -que se dio solo en el $30 \%$ de los niños- y los síntomas gastrointestinales. Estos resultados son parecidos a los observados en otros estudios que agrupaban un número elevado de casos y estudiaban pacientes en cuarentena y enfermos no hospitalizados $^{18,19}$, así como a los de un estudio similar realizado en nuestro medio durante la epidemia gripal del año $1996^{20}$.

Nuestros casos fueron, en general, leves y prácticamente no hubo complicaciones. Ni siquiera los niños con antecedentes de asma (uno de cada cuatro de los pacientes con gripe confirmada) tuvieron cuadros de gravedad, y la mayoría no presentó crisis asmáticas. Ninguno de nuestros pacientes recibió tratamiento antiviral ya que, para cuando se recibieron en las consultas los resultados virológicos (aproximadamente a la semana de la recogida de las muestras), todos ellos refirieron una buena evolución de su cuadro gripal.

Como conclusión, destacar que, a pesar de nuestros temores iniciales, los casos de gripe pandémica A (H1N1) 2009 en los niños atendidos en nuestras consultas de AP han sido generalmente leves.

\section{Bibliografía}

1. Centers for Disease Control and Prevention (CDC). Swine Influenza A (H1N1) infections-California and Texas, April 2009. MMWR. 2009;58 (16):435-7 [consultado el 10/04/2010]. Disponible en www.cdc.gov/mmwr/preview/mmwrhtml/ mm5816a7.htm

2. Centers for Disease Control and Prevention (CDC). Outbreak of swine influenza A (H1N1) virus infection-Mexico, March-April 2009. MMWR. 2009;58(Dispactch):1-3 [consultado el 10/04/ 2010]. Disponible en www.cdc.gov/mmwr/ preview/mmwrhtml/mm58d0430a2.htm

3. Organización Mundial de la Salud. Declaración de la Directora General de la OMS a la prensa [publicado el 11/06/2009] [consultado el 10/04/ 2010]. Disponible en www.who.int/mediacen tre/news/statements/2009/h1n1_pandemic _ phase6_20090611/es/index.html
4. Generalitat de Catalunya. Departament de Salut. Pla d'informació de les infeccions respiratòries agudes a Catalunya (PIDIRAC) [consultado el 10/04/2010]. Disponible en www.gencat.cat/ salut/depsalut/html/ca/dir3157/plapidirac.pdf

5. Generalitat de Catalunya. Departament de Salut. Pla d'actuació a Catalunya enfront d'una infecció pel virus pandèmic (H1N1) 2009 [consultado el 12/09/2009]. Disponible en www.gen cat.cat/salut/depsalut/html/ca/dir438/gr2704200 9.pdf

6. OPS-CDC. PAHO/HDM/CD/V/411/06. Protocolo genérico para la vigilancia de la influenza [consultado el 12/09/2009]. Disponible en http:// new. paho.org/hq/index.php?option=com docman\&task=doc_download\&gid=2080\&ltemid=

7. Red Nacional de Vigilancia Epidemiológica. Mantenimiento de la vigilancia de gripe estacional a través del sistema de vigilancia de gripe en España. Fecha: 14.07.09 [consultado el 12/09/2009]. 
Disponible en www.msc.es/profesionales/salud Publica/gripeA/docs/ANEXOIProtocolo_Manteni mientoVigilanciaGripe_SVGE_v4_140709.pdf

8. Generalitat de Catalunya. Pla d'informació de les infeccions respiratòries agudes a Catalunya (PIDIRAC). Temporada gripal 2009-2010. Full informatiu núm.33 (26.05.10) [consultado el 10/06/ 2010] Disponible en www.gencat.cat/salut/ depsalut/html/ca/dir3157/pidirac_2009_10.pdf

9. Potter CW. A history of influenza. J Appl Microbiol. 2001;91:572-9.

10. Gobierno Vasco. Plan de acción frente a una pandemia gripal en Euskadi. Adaptación y puesta al día del documento aprobado en 2006 por la Comisión Interdepartamental para la elaboración y desarrollo de un Plan de Acción frente a una Pandemia Gripal. 2009 Sep [consultado el 10/06/ 2010]. Disponible en www.osanet.euskadi.net/ r85-gripe02/05/contenidos/informacion/gripe plan/es_gripe/adjuntos/plan.pdf

11. Otero S, Moraga JA. Desarrollo y autorización de vacunas pandémicas de la gripe A (H1N1) 2009. Vacunas. 2010;11:17-24.

12. Organización Mundial de la Salud. Summary table of SARS cases by country, 1 November 2002-7 August 2003 [consultado el 22/04/2010]. Disponible en www.who.int/csr/sars/country/ country2003_08_15.pdf

13. Caylà JA. Epidemias mediáticas: una reflexión para la salud pública. Gac Sanit. 2009;23:3624 [consultado el 10/06/2010]. Disponible en http://scielo.isciii.es/scielo.php?script=sci_art text\&pid=S0213-91112009000500002\&lng=es
14. Baker MG, Kelly $H$, Wilson N. Pandemic H1N1 influenza lessons from the southern hemisphere. Euro Surveill. 2009;14(42):pii=19370 [consultado el 14/05/2010]. Disponible en www.cdc. gov/flu/weekly/pdf/External_F0952.pdf

15. Cao B, Li XW, Mao Y, Wang J, Lu HZ, Chen $Y S$, et al. Clinical features of the initial cases of 2009 pandemic influenza $A(H 1 N 1)$ virus infection in China. N Engl J Med. 2009;361:2507-17.

16. Kamigaki T, Oshitani H. Epidemiological characteristics and low case fatality rate of pandemic (H1N1) 2009 in Japan. PLoS Curr Influenza. 2009;20:RRN1139.

17. Morgan OW, Parks S, Shim T, Blevins PA, Lucas PM, Sanchez R, et al. Household transmission of pandemic (H1N1) 2009, San Antonio, Texas, USA, April-May 2009. Emerg Infect Dis. 2010;16:631-7.

18. Clinical Aspects of Pandemic 2009 Influenza A (H1N1) Virus Infection. Writing Committee of the WHO Consultation on Clinical Aspects of Pandemic (H1N1) 2009 Influenza. N Engl J Med. 2010;362:1708-19.

19. Cowling BJ, Chan KH, Fang VJ, Lau LL, So $\mathrm{HC}$, Fung RO, et al. Comparative epidemiology of pandemic and seasonal influenza $A$ in households. N Engl J Med. 2010;362:2175-84.

20. Van Esso DL, Estabanell $A$, Fernández I, Pérez M, Besora R, Casanovas JM, et al. Aspectos clínico-epidemiológicos de la infección por virus de la gripe $A$ en menores de 7 años en atención primaria. An Pediatr (Barc). 2006;65: 211-8. 